\title{
Radiologic Response as a Prognostic Factor in Advanced Hepatocellular Carcinoma with Macroscopic Vascular Invasion after Transarterial Chemoembolization and Radiotherapy
}

\author{
Jinhong Jung ${ }^{\mathrm{a}}$ Ji Hyeon Joo ${ }^{\mathrm{a}}$ So Yeon Kim ${ }^{\mathrm{b}}$ Jin Hyoung Kim ${ }^{\mathrm{b}}$ Jonggi Choic \\ Danbi Lee ${ }^{c}$ Ju Hyun Shim ${ }^{c}$ Kang Mo Kimc Young-Suk Limc Han Chu Lee ${ }^{c}$ \\ Jin-hong Park ${ }^{a}$ Sang Min Yoon ${ }^{a}$ \\ aDepartment of Radiation Oncology, Asan Liver Center, Asan Medical Center, University of Ulsan College of \\ Medicine, Seoul, Republic of Korea; 'bepartment of Radiology and the Research Institute of Radiology, Asan Liver \\ Center, Asan Medical Center, University of Ulsan College of Medicine, Seoul, Republic of Korea; ' ${ }^{\mathrm{D}}$ epartment of \\ Gastroenterology, Asan Liver Center, Asan Medical Center, University of Ulsan College of Medicine, Seoul, Republic \\ of Korea
}

\section{Keywords}

Hepatocellular carcinoma · Vascular invasion · Radiotherapy ·

Transarterial chemoembolization · Response

\begin{abstract}
Introduction: We evaluated the radiologic response rate of combined transarterial chemoembolization (TACE) plus radiotherapy (RT) in treatment-naïve patients with liver-confined hepatocellular carcinoma (HCC) with macroscopic vascular invasion (MVI) and analyzed its clinical importance in overall survival (OS) outcomes. Methods: Patients who were treated with TACE plus RT as a first-line treatment for HCC with MVI between January 2010 and December 2015 were retrospectively reviewed. Radiologic response was assessed according to the modified Response Evaluation Criteria in Solid Tumors (mRECIST) at 2 and 4 months after completion of RT. Landmark analysis at 2 and 4 months, and time-dependent Cox regression analysis using response as a time-dependent covariate were performed for univariable and multivariable analyses. Results: The 2-month landmark analysis
\end{abstract}

karger@karger.com www.karger.com/lic

Karger!"

GOPEN ACCESS
(C) 2021 The Author(s)

Published by S. Karger AG, Basel

This is an Open Access article licensed under the Creative Commons Attribution-NonCommercial-4.0 International License (CC BY-NC) (http://www.karger.com/Services/OpenAccessLicense), applicable to the online version of the article only. Usage and distribution for commercial purposes requires written permission. included 427 patients, and the 4-month landmark analysis included 355 patients after excluding patients without imaging studies for response evaluation at 4 months. Radiologic responses were observed in 210 (49.2\%) patients at 2 months and $181(51.8 \%)$ patients at 4 months. In multivariable analyses, radiologic response was identified as an independent prognosticator for OS at 2 months (median OS: responders, 23.1 months vs. nonresponders, 8.0 months; hazard ratio $[H R], 3.194 ; p<0.001$ ) and 4 months (median OS: responders, 26.5 months vs. nonresponders, 9.3 months; HR, 4.534; $p<0.001$ ). Conclusion: Radiologic response assessed by $\mathrm{mRECIST}$ was a significant prognostic factor for OS in patients with advanced-stage HCC showing MVI treated with combined TACE plus RT.

(C) 2021 The Author(s)

Published by S. Karger AG, Basel

Ji Hyeon Joo: Department of Radiation Oncology, Pusan National University Yangsan Hospital, Yangsan, Republic of Korea.

Jinhong Jung and Ji Hyeon Joo contributed equally to this work as co-first authors.
Correspondence to:

Sang Min Yoon, drsmyoon@amc.seoul.kr 


\section{Introduction}

Liver cancer is the fourth leading cause of cancer death worldwide and the second leading cause of cancer death in males [1]. Because $75-85 \%$ of cases of liver cancers are hepatocellular carcinoma (HCC) [1], this mortality rate is closely related to the prognosis of HCC. Despite the efforts for developing the prevention and surveillance strategies for HCC, the majority of patients with HCC present with locally advanced disease showing macroscopic vascular invasion (MVI) into the portal vein, hepatic vein, and inferior vena cava (IVC) [2]. In the current treatment guidelines, the standard of care in advanced-stage HCC (i.e., the Barcelona Clinic Liver Cancer [BCLC] stage C) is systemic therapies [3, 4], and sorafenib and lenvatinib have been used as first-line systemic therapies for advanced-stage HCCs during the last decade [5-7]. However, the presence of MVI is associated with a worse prognosis in patients with BCLC stage $\mathrm{C}$ disease, and both sorafenib and lenvatinib showed lower survival outcomes in subgroups of patients with MVI [7, 8]. Recently, the combination of atezolizumab and bevacizumab showed promising efficacy in patients with unresectable HCC and was regarded as an alternative standard of care in advanced-stage HCC [9]; however, further information regarding the efficacy of this combination regimen in patients with HCC and MVI is needed.

Vascular invasion is one of the main routes of disease spreading from the primary tumor site as well as an important factor for the deterioration of hepatic function. Considering the major contribution of MVI to poor prognosis, achievement of early response and higher response rate may be a priority in selecting the optimal initial treatments $[10,11]$. In this respect, external beam radiotherapy (RT) combined with locoregional treatments has been widely used for HCC patients with MVI [12]. A recent randomized clinical trial demonstrated that compared with sorafenib, combined transarterial chemoembolization (TACE) and RT yielded a significantly higher response rate and subsequently a longer overall survival (OS) rate [13].

Considering such promising results, there is a need for a large-sized study evaluating the clinical significance of the radiologic response rate after TACE plus RT as a firstline treatment for HCC patients with MVI. In the present study, we evaluated the radiologic response rate of combined TACE plus RT for treatment-naïve, liver-confined HCC patients with MVI, and analyzed its clinical impact in terms of OS.

Early Response after TACE plus RT for HCC with MVI

\section{Materials and Methods}

\section{Patients}

We reviewed the medical records of HCC patients with MVI who were treated with combined TACE and respiratory-gated 3-dimensional conformal RT as a first-line treatment between January 2010 and December 2015 at Asan Medical Center (Seoul, Republic of Korea). The eligibility criteria were similar to those of our previous study [14], except for the exclusion of patients with extrahepatic metastases at diagnosis: (a) preserved hepatic function (i.e., Child-Pugh classification A or B); (b) European Cooperative Oncology Group (ECOG) performance status of $0-2$; (c) no evidence of extrahepatic nodal or visceral metastases; and (d) no history of previous treatments for HCC. Patients were excluded if they had uncontrolled ascites, hepatic encephalopathy, an active gastric or duodenal ulcer, other uncontrolled comorbidities, or double primary malignant tumors. Patients who did not undergo imaging studies for response evaluation were also excluded from this study.

$\mathrm{HCC}$ was diagnosed on the basis of pathologic findings and/or typical features on multi-phase dynamic computed tomography (CT) or magnetic resonance images using a hepatocyte-specific contrast agent according to the American Association for the Study of Liver Diseases criteria [15]. The presence of MVI was assessed with the following criteria: (a) intraluminal filling defect adjacent to the HCC in the portal vein, hepatic vein, and/or IVC, and (b) enhancement of the filling defect on the arterial phase and washout on the portal/delayed phases [13]. This study was approved by the Institutional Review Board of Asan Medical Center (\#2017-0491), and the requirement for informed consent was waived considering the retrospective nature of the present study.

\section{Transarterial Chemoembolization}

The TACE procedure was described in our previous studies [10, $14,16,17]$. Cisplatin-based TACE with $2 \mathrm{mg} / \mathrm{kg}$ of cisplatin (Dong-A Pharmaceutical, Seosan, Republic of Korea) was performed using a microcatheter placed directly into the feeding artery. Embolization was performed using an emulsion of 3-20 mL of iodized oil (Lipiodol; Guerbet, Roissy, France) and cisplatin mixture, which was followed by embolization with gelfoam slurry (Upjohn, Kalamazoo, MI, USA). Transarterial chemotherapeutic infusion (TACI) without gelfoam slurry was performed according to the severity of portal vein blood flow impairment. In general, TACI was performed in patients with (1) main portal vein tumor thrombosis with poorly developed collaterals, or (2) lobar or segmental portal vein tumor thrombosis with HCCs occupying more than one-half of the liver. All HCCs were treated in a single TACE or TACI session. TACE or TACI was performed before or after RT within an interval of 4 weeks and was repeated every 6-8 weeks in considering the residual viable HCCs without deterioration of hepatic function $[14,17]$.

\section{External Beam Radiotherapy}

The detailed procedures of RT were described in our previous studies [14, 17]. Four-dimensional (4D) CT (GE LightSpeed RT 16; GE Healthcare, Waukesha, WI, USA) images were acquired from all patients with free breathing. The patients' breathing patterns were analyzed by the Real-time Position Management respiratory gating system (Varian Medical Systems, Palo Alto, CA, USA), and all CT datasets were sorted into 10 phase bins using a 4D imaging software (Advantage 4D; GE Healthcare). Target volumes were delineated in the end-exhale phase CT images. In most 


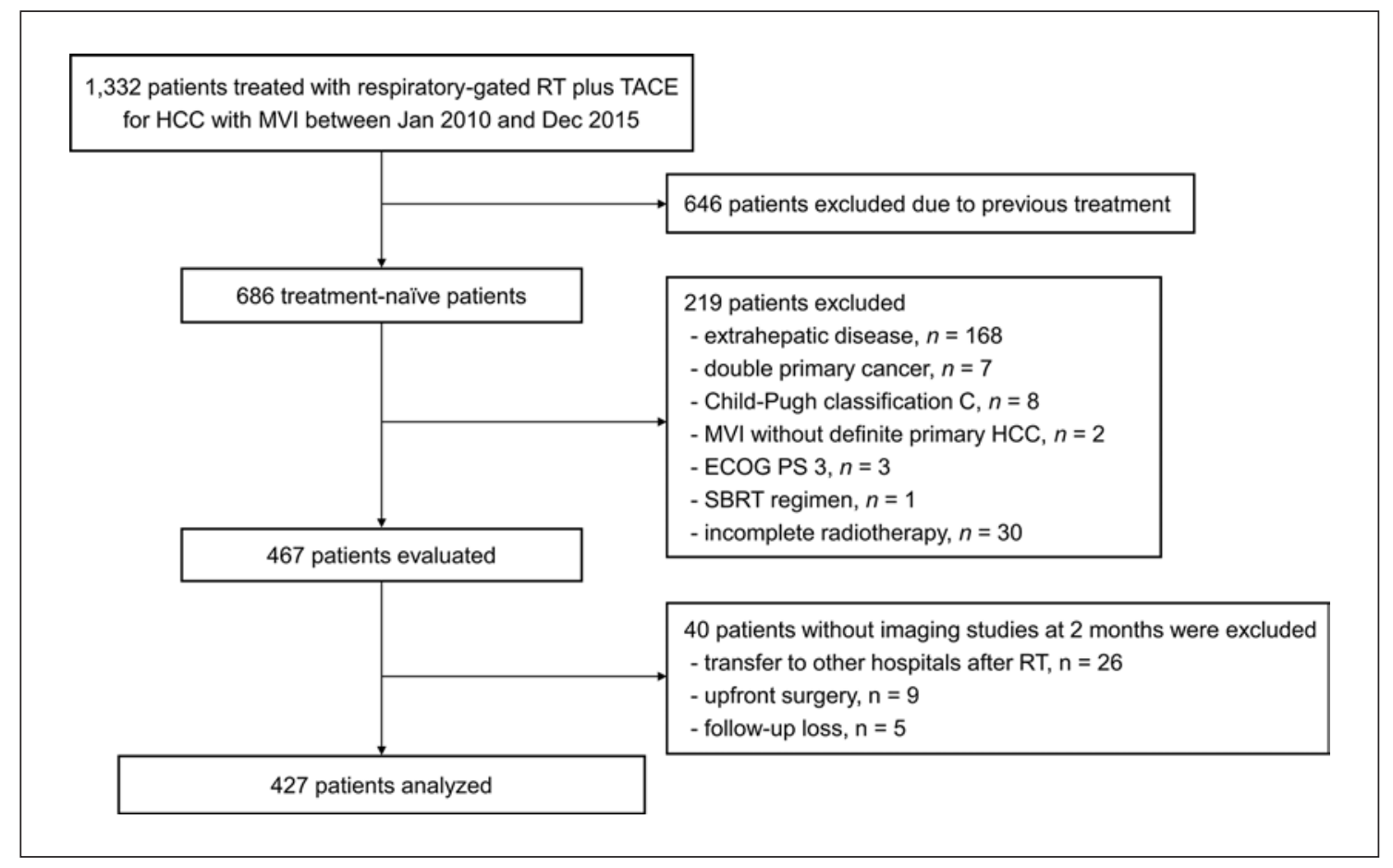

Fig. 1. Study population flowchart. SBRT, stereotactic body radiotherapy.

patients with a large, infiltrative HCC, the gross tumor volume (GTV) included the MVI and a 2-cm expansion into the contiguous HCC. In cases of small HCCs, both the entire HCC and MVI were included in the GTV. In select cases of extensive portal vein invasion with a Child-Pugh B liver function, the GTV included the MVI itself without including HCC in the liver parenchyma [14, 17]. The internal target volume was generated as the sum of the individual GTVs as defined within the gated phases of respiration. A margin of $0.7 \mathrm{~cm}$ from the internal target volume was applied for the planning target volume $[14,17]$.

The dose per fraction to the planning target volume was $2-5 \mathrm{~Gy}$ using 6- or 15-MV X-rays at 5 fractions per week using linear accelerators (Varian Medical Systems). The total dose was determined by the volume of normal liver, liver function, and the maximum dose to the organs at risk. The general guideline was that the fraction of the normal liver treated with more than $50 \%$ of the prescribed dose should be less than $50 \%$ of the normal liver volume $\left(\mathrm{V}_{50 \%}<50 \%\right)$. The volume of the normal liver that was damaged by irradiation was defined as the fraction volume of the normal liver that received more than $30 \mathrm{~Gy}\left(\mathrm{~V}_{30 \mathrm{~Gy}}\right)$, with no more than $30 \%$ of the normal liver being exposed to more than $30 \mathrm{~Gy}\left(\mathrm{~V}_{30 \mathrm{~Gy}} \leq 30 \%\right)$ [10]. The three-dimensional conformal RT technique was performed using an RT planning system (Eclipse version 10.0; Varian Medical Systems), and actual beam delivery was performed with an image-guided, respiratory-gated RT technique [14].

\section{Evaluation}

Radiologic response was assessed by considering the target and nontarget lesions (i.e., overall response) according to the modified Response Evaluation Criteria in Solid Tumors (mRECIST) at 2 fixed time points ( 2 and 4 months) after the completion of RT using dy- namic CT or magnetic resonance images [18]. The best response during treatment was also evaluated according to the mRECIST on available follow-up images. Tumor measurements and response evaluation were carried out by an abdominal radiologist with 15 years of experience in HCC imaging and a radiation oncologist with 8 years of experience in RT for HCC. Radiologic reviews were performed independently, and a consensus decision was made together in case of a discrepancy in interpretation with the third additional reviewer (a radiation oncologist with 15 years of experience in RT for HCC). Responders were defined as patients who had a complete response (CR) or partial response (PR). Adverse events associated with RT were analyzed using the Common Terminology Criteria for Adverse Events version 4.03. Nonclassic radiation-induced liver disease was also graded according to the Common Terminology Criteria for Adverse Events or worsening of the Child-Pugh score by 2 or more in the absence of progressive disease within 3 months after RT [19].

\section{Statistical Analysis}

Patients were categorized into 2 groups (responders vs. nonresponders) according to the best response. Baseline characteristics were compared between the 2 groups using the $\chi^{2}$ test for categorical variables, and the Student's $t$ test or Mann-Whitney U test for continuous variables. The rates of OS and progression-free survival (PFS) were estimated from the date of treatment initiation for HCC to the date of death, the last follow-up, or the date of tumor progression. The probability of cumulative survival was calculated using the Kaplan-Meier method. The status of radiologic response to treatment changed during the follow-up period. Because the OS rate might be underestimated in nonresponders and overestimated in responders, leading to guarantee-time bias [20], we performed landmark analyses at 2 fixed time points ( 2 and 4 months 
Table 1. Baseline characteristics of patients according to treatment response

\begin{tabular}{|c|c|c|c|c|}
\hline Variables & $\begin{array}{l}\text { All patients } \\
(n=427)\end{array}$ & $\begin{array}{l}\text { Responders } \\
(n=233)\end{array}$ & $\begin{array}{l}\text { Nonresponders } \\
(n=194)\end{array}$ & $p$ value \\
\hline Male sex & $380(89.0)$ & $207(88.8)$ & $173(89.2)$ & $>0.99$ \\
\hline Age, years & $57(34-87)$ & $58(37-87)$ & $57(34-84)$ & 0.12 \\
\hline \multicolumn{5}{|l|}{ ECOG performance status } \\
\hline 0 & $123(28.8)$ & $80(34.3)$ & $43(22.2)$ & \multirow{2}{*}{0.008} \\
\hline $1-2$ & $304(71.2)$ & $153(65.7)$ & $151(77.8)$ & \\
\hline \multicolumn{5}{|l|}{ Child-Pugh class } \\
\hline$A$ & $276(64.6)$ & $169(72.5)$ & $107(55.2)$ & \multirow{2}{*}{$<0.001$} \\
\hline B & $151(35.4)$ & $64(27.5)$ & $87(44.8)$ & \\
\hline \multicolumn{5}{|l|}{ Viral etiology } \\
\hline Hepatitis B virus & $371(86.9)$ & $197(84.5)$ & $174(89.7)$ & \multirow{3}{*}{0.21} \\
\hline Hepatitis $C$ virus & $13(3.0)$ & $10(4.3)$ & $3(1.5)$ & \\
\hline Non-B, non-C & $43(10.1)$ & $26(11.2)$ & $17(8.8)$ & \\
\hline \multicolumn{5}{|l|}{ Tumor multiplicity } \\
\hline Single & $125(29.3)$ & $83(35.6)$ & $42(21.6)$ & \multirow{2}{*}{0.002} \\
\hline Multiple & $302(70.7)$ & $150(64.4)$ & $152(78.4)$ & \\
\hline \multicolumn{5}{|l|}{ Extent of the tumor } \\
\hline Unilobar & $223(52.2)$ & $131(56.2)$ & $92(47.4)$ & \multirow{2}{*}{0.09} \\
\hline Bilobar & $204(47.8)$ & $102(43.8)$ & $102(52.6)$ & \\
\hline \multicolumn{5}{|l|}{ Tumor type } \\
\hline Nodular & $69(16.2)$ & $50(21.5)$ & $19(9.8)$ & \multirow{2}{*}{0.002} \\
\hline Infiltrative & $358(83.8)$ & $183(78.5)$ & $175(90.2)$ & \\
\hline Size of the largest lesion, $\mathrm{cm}$ & \multirow{2}{*}{\multicolumn{4}{|c|}{ Extent of vascular invasion }} \\
\hline Unilateral PV & $186(43.6)$ & $122(52.6)$ & $64(32.8)$ & \\
\hline Bilateral/main PV, IVC & $241(56.4)$ & $111(47.6)$ & $130(67.0)$ & $<0.001$ \\
\hline Bile duct invasion & $32(7.5)$ & $14(6.0)$ & $18(9.3)$ & 0.27 \\
\hline Alpha-fetoprotein, $\mathrm{ng} / \mathrm{mL}$ & $1,424.5(1.6-3,132,070)$ & $1,229.3(1.6-628,000)$ & $1,575.2(1.7-3,132,070)$ & 0.042 \\
\hline PIVKA-II, mAU/mL ${ }^{\mathrm{b}}$ & $3,332(10-75,000)$ & $1,720.5(10-75,000)$ & $6,791.5(23-75,000)$ & $<0.001$ \\
\hline
\end{tabular}

Values are $n$ (\%) or median (range). ECOG, Eastern Cooperative Oncology Group; PV, portal vein; IVC, inferior vena cava; PIVKA, protein induced by vitamin K absence or antagonist; mRECIST, modified response evaluation criteria in solid tumors. ${ }^{a}$ Response was defined as the best response during treatment according to the mRECIST. b PIVKA-Il examinations were conducted in 416 patients (226 responders and 190 nonresponders).

after completion of RT). In addition, Cox regression analysis using treatment response as a time-dependent covariate was performed to describe the associations of variables with OS, and the hazard ratios (HRs) and their confidence intervals (CIs) in univariable and multivariable analyses were computed. Variables significantly associated with OS $(p \leq 0.1)$ in univariable analysis were chosen for multivariable analysis using backward elimination. Two-sided $p$ values $<0.05$ were considered statistically significant. All statistical analyses were performed using $\mathrm{R}$ version 4.0.2 ( $\mathrm{R}$ Foundation for Statistical Computing, Vienna, Austria; http://cran.r-project.org).

\section{Results}

\section{Patient Characteristics}

Out of 1,332 patients who received combined TACE plus RT for HCC with MVI during the study period, a total of 467 patients were evaluated. Of them, 40 patients without imaging studies at 2 months were excluded due to the following reasons: transfer to other hospitals closer to their residence after RT $(n=26)$, upfront surgery $(n=9)$, and follow-up loss $(n=5)$. Finally, 427 patients were included in the 2-month landmark analysis. After excluding 72 patients without imaging studies for response evaluation at 4 months, the 4-month landmark analysis included a total of 355 patients (Fig. 1). The baseline characteristics of the patients according to the treatment response are shown in Table 1 . The median age of the patients as a whole was 57 years (range, $34-87$ ), and $89.0 \%$ were male. The median value of the maximal diameter of the HCCs was $9.6 \mathrm{~cm}$ (range, 1.1-21.1), and $276(64.6 \%)$ patients had a hepatic function of Child-Pugh class A. Most of the patients had multiple lesions $(70.7 \%)$ and infiltrative type HCC (83.8\%). 
Table 2. Radiologic response rates after TACE and RT according to evaluation timing

\begin{tabular}{llll}
\hline & At 2 months & At 4 months ${ }^{\mathrm{b}}$ & Best response \\
\hline Objective response rate, ${ }^{\mathrm{a}} n(\%)$ & $210(49.2)$ & $184(51.8)$ & $233(54.6)$ \\
CR & $39(9.1)$ & $67(18.9)$ & $96(22.5)$ \\
PR & $171(40.1)$ & $117(32.9)$ & $137(32.1)$ \\
Stable disease & $106(24.8)$ & $30(8.5)$ & $83(19.4)$ \\
Progressive disease & $111(26.0)$ & $141(39.7)$ & $111(26.0)$ \\
\hline
\end{tabular}

TACE, transarterial chemoembolization; RT, radiotherapy; CR, complete response; PR, partial response. ${ }^{\text {a }}$ Proportion of patients who had CR or PR. ${ }^{b} n=355$ patients who had evaluable response at 4 months after treatment.

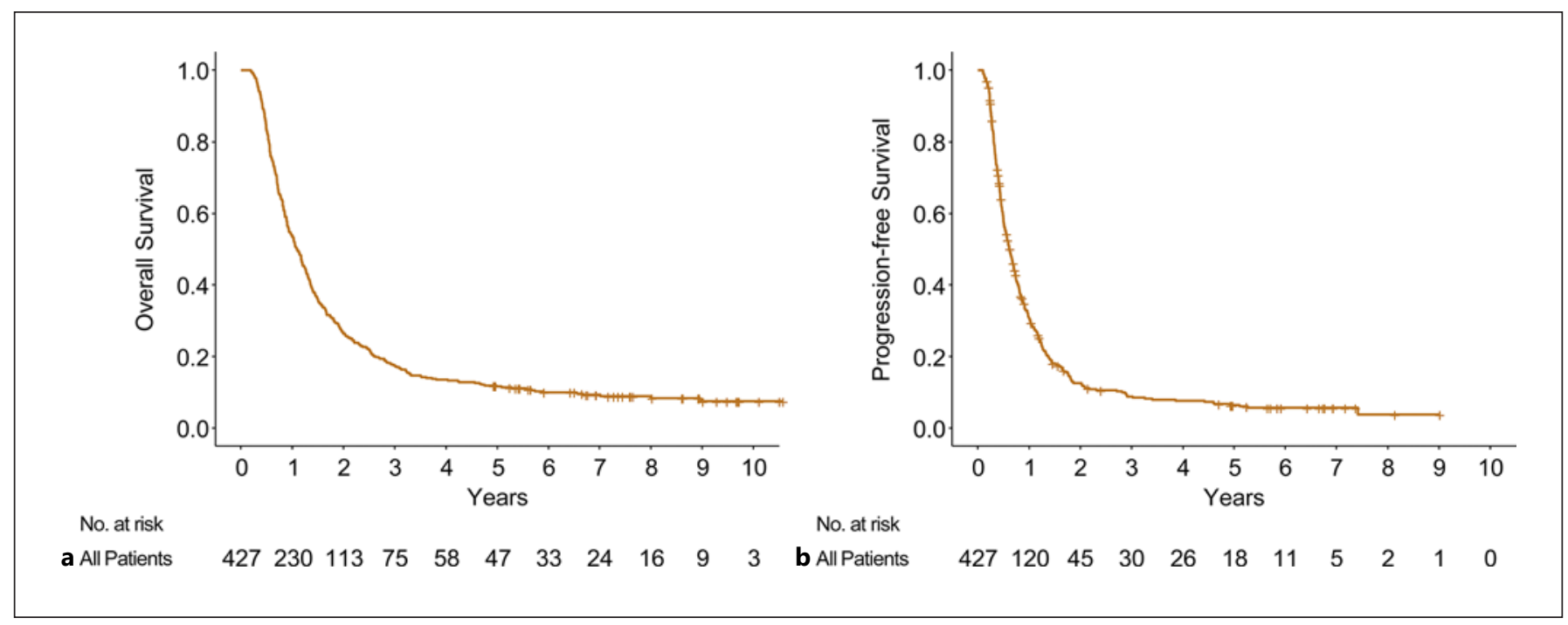

Fig. 2. Survival rates of the total patients. a OS (median, 13.1 months [95\% CI, 11.6-14.9]). b PFS (median, 7.4 months [95\% CI, 6.6-8.4]).

More than half of the patients (56.4\%) were diagnosed with main/bilateral portal vein and/or IVC invasion.

When divided according to treatment response, the responder group had a significantly higher proportion of patients with good hepatic function $(p<0.001)$, good performance status $(p=0.008)$, single tumor $(p=0.002)$, nodular tumor type $(p=0.002)$, smaller tumor size $(p<$ $0.001)$, unilateral vascular involvement $(p<0.001)$, and lower tumor marker levels (alpha-fetoprotein, $p=0.042$; protein induced by vitamin $\mathrm{K}$ absence or antagonist-II, $p<0.001$ ). The median radiation dose was $40 \mathrm{~Gy}$ (range, $24-50$ ), and the median fraction size was 2.5 Gy (range, 2-5) (online suppl. Table 1; for all online suppl. material, see www.karger.com/doi/10.1159/000521227). Due to the reduced portal blood flow by tumor thrombus, fulldose chemoembolization (i.e., with cisplatin, iodized oil, and gelfoam slurry) was performed in $372(87.1 \%)$ patients for the first TACE. Repeated TACE after RT was performed for residual viable HCCs (median, 3), and the number of repeated TACE was significantly higher in patients with Child-Pugh A hepatic function (online suppl. Tables 1,2). Subsequent systemic therapy was applied in 167 (39.1\%) patients when progressive intrahepatic HCCs and/or extrahepatic metastases were found after repeated TACE (online suppl. Tables 1,2).

\section{Clinical Outcomes}

The median follow-up duration of the total patients was 13.1 months (range, 2.3-126.8). By the last follow-up, 39 (9.1\%) patients were alive; the rates of 1-, 2-, and 5-year OS were $53.9 \%, 26.5 \%$, and $11.7 \%$, respectively, and the median OS duration was 13.1 months (95\% CI, 11.614.9) (Fig. 2a). The median duration of PFS was 7.4 months (95\% CI, 6.6-8.4) (Fig. 2b).

No patients experienced acute grade $\geq 3$ constitutional symptoms after RT (online suppl. Table 3 ). Within 3 
Table 3. Univariable and multivariable analyses for prognostic factors on OS rates at the 2-month landmark

\begin{tabular}{|c|c|c|c|c|}
\hline \multirow[t]{2}{*}{ Variables } & \multicolumn{2}{|l|}{ Univariable } & \multicolumn{2}{|l|}{ Multivariable } \\
\hline & $\mathrm{HR}(95 \% \mathrm{Cl})$ & $p$ value & $\mathrm{HR}(95 \% \mathrm{Cl})$ & $p$ value \\
\hline Male (female) & $1.178(0.841-1.650)$ & 0.34 & & \\
\hline Age & $0.999(0.988-1.010)$ & 0.82 & & \\
\hline ECOG performance status (0) & $1.445(1.160-1.800)$ & 0.001 & $1.241(0.969-1.588)$ & 0.09 \\
\hline Child-Pugh class (A) & $1.945(1.563-2.419)$ & $<0.001$ & $1.388(1.031-1.869)$ & 0.031 \\
\hline Viral etiology $(\mathrm{HBV})^{\mathrm{a}}$ & $0.916(0.697-1.203)$ & 0.53 & & \\
\hline Multiplicity (single) & $1.458(1.172-1.813)$ & 0.001 & $1.388(1.068-1.804)$ & 0.014 \\
\hline Extent of the tumor (unilobar) & $1.364(1.118-1.664)$ & 0.002 & $1.168(0.904-1.510)$ & 0.24 \\
\hline Tumor type (nodular) & $1.626(1.238-2.136)$ & $<0.001$ & $1.277(0.955-1.706)$ & 0.10 \\
\hline Tumor size & $1.097(1.070-1.125)$ & $<0.001$ & $1.035(1.001-1.070)$ & 0.046 \\
\hline Extent of vascular invasion (unilateral PV) & $1.468(1.202-1.793)$ & $<0.001$ & $0.866(0.674-1.112)$ & 0.26 \\
\hline Bile duct invasion (none) & $1.020(0.654-1.588)$ & 0.93 & & \\
\hline Alpha-fetoprotein ( $\leq 400$ ng/mL) & $1.156(0.941-1.420)$ & 0.17 & & \\
\hline PIVKA-II $(\leq 3,000 \mathrm{mAU} / \mathrm{mL})$ & $1.294(1.058-1.582)$ & 0.012 & $0.819(0.649-1.034)$ & 0.09 \\
\hline Radiotherapy dose & $0.971(0.954-0.989)$ & 0.001 & $0.981(0.961-1.002)$ & 0.07 \\
\hline Subsequent systemic therapy (yes) & $0.994(0.826-1.195)$ & 0.947 & & \\
\hline Distant metastasis (none) & $1.313(1.048-1.645)$ & 0.018 & $0.977(0.771-1.238)$ & 0.84 \\
\hline Intrahepatic progression (none) & $1.631(1.177-2.260)$ & 0.003 & $1.531(1.052-2.226)$ & 0.026 \\
\hline Radiologic response at 2 months (responder) & $4.045(3.161-5.177)$ & $<0.001$ & $3.194(2.455-4.156)$ & $<0.001$ \\
\hline
\end{tabular}

ECOG, Eastern Cooperative Oncology Group; HBV, hepatitis B virus; PV, portal vein; PIVKA, protein induced by vitamin $\mathrm{K}$ absence or antagonist; $\mathrm{HR}$, hazard ratio; $\mathrm{Cl}$, confidence interval; OS, overall survival. Values in the paren-

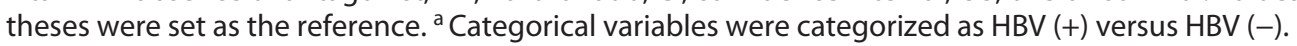

months after the completion of RT, grade $\geq 3$ liver enzyme elevation and bilirubin increase were observed in 31 $(8.2 \%)$ and $6(1.4 \%)$ patients, respectively. Compared with the baseline Child-Pugh score, 64 (16.8\%) patients experienced worsening of the Child-Pugh score by $\geq 2$; however, hepatic failure without intrahepatic progression was observed in $3(0.7 \%)$ patients. As for the late toxicities of RT, grade $\geq 3$ gastrointestinal bleeding occurred in 9 (2.1\%) patients during follow-up.

Radiologic Response as an Independent Prognosticator

At 2 months after the completion of RT, radiologic responses were observed in $210(49.2 \%)$ patients (CR, 39 [9.1\%]; PR, 171 [40.1\%]) (Table 2). At 4 months, 184 (51.8\%) patients among 355 evaluable patients showed radiologic response (CR, 67 [18.9\%]; PR, 117 [32.9\%]). The best response during treatment was observed in 233 (54.6\%) patients (Table 2; online suppl. Table 1).

The landmark analysis at 2 months revealed that the OS was significantly associated with ECOG performance status, Child-Pugh class, multiplicity, tumor extent, tumor type, tumor size, extent of vascular invasion, protein induced by vitamin $\mathrm{K}$ absence or antagonist-II, radiation dose, distant metastasis, intrahepatic progression, and radiologic response at 2 months (Table 3). Multivariable time-dependent Cox regression analysis showed that radiologic response at 2 months was an independent prognostic factor of OS (HR, 3.194; 95\% CI, 2.455-4.156, $p<0.001)$ along with Child-Pugh class $(p=0.031)$, multiplicity $(p=0.014)$, tumor size $(p=0.046)$, and intrahepatic progression $(p=0.026)$. The median OS of the 2-month responders was 23.1 months (95\% CI, 20.1-26.6), which was significantly longer than the 8.0 months median OS (95\% CI: 6.98.7) in nonresponders (Fig. 3a).

Similarly, landmark analysis at 4 months showed that the OS was significantly associated with ECOG performance status, Child-Pugh class, multiplicity, tumor extent, tumor type, tumor size, extent of vascular invasion, radiation dose, distant metastasis, intrahepatic progression, and radiologic response at 4 months (Table 4). In multivariable analysis, radiologic response at 4 months (HR, 4.534; 95\% CI, 3.391-6.062, $p<0.001$ ) was an independent prognosticator for OS along with ECOG performance status $(p=0.020)$, Child-Pugh class $(p=0.012)$, multiplicity $(p=0.005)$, and intrahepatic progression $(p=0.038)$. The median OS of the 4-month responders was significantly longer (26.5 months; 95\% CI, 22.8-31.5) 
Table 4. Univariable and multivariable analyses for prognostic factors on OS rates at the 4-month landmark

\begin{tabular}{|c|c|c|c|c|}
\hline \multirow[t]{2}{*}{ Variables } & \multicolumn{2}{|l|}{ Univariable } & \multicolumn{2}{|l|}{ Multivariable } \\
\hline & $\mathrm{HR}(95 \% \mathrm{Cl})$ & $p$ value & $\mathrm{HR}(95 \% \mathrm{Cl})$ & $p$ value \\
\hline Male (female) & $1.129(0.787-1.620)$ & 0.51 & & \\
\hline Age & $0.999(0.987-1.011)$ & 0.85 & & \\
\hline ECOG performance status (0) & $1.438(1.130-1.830)$ & 0.003 & $1.375(1.051-1.798)$ & 0.020 \\
\hline Child-Pugh class (A) & $1.729(1.356-2.206)$ & $<0.001$ & $1.477(1.091-1.999)$ & 0.012 \\
\hline Viral etiology (HBV) ${ }^{a}$ & $0.904(0.670-1.219)$ & 0.51 & & \\
\hline Multiplicity (single) & $1.502(1.181-1.910)$ & 0.001 & 1.487 (1.127-1.962) & 0.005 \\
\hline Extent of the tumor (unilobar) & $1.330(1.068-1.657)$ & 0.011 & $1.115(0.862-1.442)$ & 0.41 \\
\hline Tumor type (nodular) & $1.456(1.088-1.949)$ & 0.012 & $1.083(0.800-1.466)$ & 0.61 \\
\hline Tumor size & $1.083(1.055-1.113)$ & $<0.001$ & $1.018(0.984-1.053)$ & 0.31 \\
\hline Extent of vascular invasion (unilateral PV) & $1.378(1.105-1.718)$ & 0.004 & $0.873(0.672-1.132)$ & 0.31 \\
\hline Bile duct invasion (none) & $0.836(0.499-1.399)$ & 0.50 & & \\
\hline Alpha-fetoprotein ( $\leq 400$ ng/mL) & $1.143(0.912-1.432)$ & 0.25 & & \\
\hline PIVKA-II $(\leq 3,000 \mathrm{mAU} / \mathrm{mL})$ & $1.202(0.963-1.502)$ & 0.10 & & \\
\hline Radiotherapy dose & $0.977(0.958-0.996)$ & 0.016 & $0.982(0.961-1.003)$ & 0.10 \\
\hline Subsequent systemic therapy (yes) & $0.879(0.713-1.082)$ & 0.222 & & \\
\hline Distant metastasis (none) & $1.487(1.152-1.919)$ & 0.002 & $1.025(0.773-1.359)$ & 0.86 \\
\hline Intrahepatic progression (none) & $1.774(1.229-2.561)$ & 0.002 & $1.525(1.023-2.274)$ & 0.038 \\
\hline Radiologic response at 4 months (responder) & $4.723(3.533-6.315)$ & $<0.001$ & $4.534(3.391-6.062)$ & $<0.001$ \\
\hline
\end{tabular}

ECOG, Eastern Cooperative Oncology Group; HBV, hepatitis B virus; PV, portal vein; PIVKA, protein induced by vitamin $\mathrm{K}$ absence or antagonist; $\mathrm{HR}$, hazard ratio; $\mathrm{Cl}$, confidence interval; OS, overall survival. Values in the parentheses were set as the reference. ${ }^{\text {a }}$ Categorical variables were categorized as HBV (+) versus HBV (-).



Fig. 3. OS in mRECIST responders and nonresponders. a 2 months (responders, 23.1 months [95\% CI, 20.126.6]; nonresponders, 8.0 months [95\% CI, 6.9-8.7]). b 4 months (responders, 26.5 months [95\% CI, 22.8-31.5]; nonresponders, 9.3 months [95\% CI, 8.6-10.2]).

than that of the nonresponders (9.3 months; 95\% CI, 8.610.2) (Fig. 3b). In addition, the mRECIST response was also a significant prognostic factor in patients with both Child-Pugh class A and class B hepatic function (online suppl. Fig. 1).

\section{Discussion}

In this study, combined TACE plus RT as a first-line treatment for patients with advanced-stage HCC with MVI yielded an early response rate of $49.2 \%$ at 2 months 
after RT and a high rate of best response (54.6\%). Notably, the mRECIST response was significantly associated with better survival and had the highest HR among the prognostic variables; accordingly, the responders had a longer survival duration than nonresponders in the landmark analyses at 2 and 4 months after the completion of treatment and time-dependent Cox regression analysis using treatment response as a time-dependent covariate. Our results suggest that mRECIST response has clinically important implications and is a potentially reliable surrogate for predicting the survival outcomes in HCC patients with poor prognosis at a relatively early timing of treatments.

Radiologic response assessment has been considered as an indispensable procedure in determining the therapeutic efficacy and has been used as a surrogate endpoint of survival outcomes. mRECIST has been proposed for use as standard response criteria for HCC after locoregional therapy $[3,4]$, and several studies have demonstrated the prognostic relevance of objective response by mRECIST in HCC patients treated with TACE $[21,22]$. In a recent meta-analysis, objective response according to mRECIST showed a strong prognostic value in terms of OS in HCC patients who received locoregional treatments [23]. In addition, analyses of data from prospective trials provided evidence to support the mRECIST response as a reliable surrogate in advanced-stage HCC after systemic therapies [24-26]. Although objective responses were observed in relatively small proportions of patients (range, 11.5-18.8\%) who received a single targeted therapy (sorafenib, nintedanib, or brivanib), objective response by mRECIST was shown to be an independent predictive and prognostic factor of OS in advancedstage HCC patients [24-26]. Especially, Lencioni et al. [26] and Kudo et al. [24] reduced the guarantee-time bias of their results by using a landmark analysis, the MantelByar test, and time-dependent analysis; these results are more reliable than the survival analysis by response based on the conventional method of responder analysis. Therefore, radiologic response can be considered as a candidate surrogate endpoint at a relatively early timing after treatment initiation in patients with advanced-stage HCC.

Among BCLC stage $\mathrm{C}$ cases, radiologic response confers a notable clinical significance for those with MVI. Portal vein invasion leads to an extensive degree of intra- or extrahepatic tumor dissemination through blood vessels as well as reductions in the blood flow to the healthy liver, thereby resulting in portal hypertension and subsequent hepatic encephalopathy, variceal bleeding, ascites, and decline in hepatic function $[2,10]$. He-

Early Response after TACE plus RT for HCC with MVI patic vein invasion usually spreads to the IVC and/or right atrium, and may lead to lung metastasis, heart failure, and even death from pulmonary thromboembolism if the tumor thrombus is not aptly managed [27]. Considering such rapid progression of tumor in such cases, timely control of MVI is crucial for delaying the functional decline of the liver and facilitating subsequent treatments $[11,13]$. In the current analysis, both intrahepatic progression and radiologic response were significant factors for OS in multivariable analyses at both time points (i.e., 2 and 4 months), which may serve as a basis for supporting our opinion. According to current guidelines, systemic treatments are the only recommended option for cases with BCLC stage C disease [3, 4]; however, one report showed that in patients with MVI, sorafenib prolonged the median survival duration by only 47 days compared with placebo (184 days vs. 137 days) [8]. The median OS after lenvatinib was also shorter in patients with MVI and/or extrahepatic metastases compared with those without MVI (11.5 months vs. 18 months) [7]. A recent study in patients with unresectable HCC showed that atezolizumab combined with bevacizumab was superior to sorafenib in terms of OS and PFS [9]; therefore, further efficacy analysis is needed for patients with MVI. In contrast, because HCC is a well-known radiosensitive tumor, many studies including a randomized clinical trial reported that the response rates in patients with HCC accompanied by MVI ranged from $30 \%$ to $70 \%$ after RT with or without combined locoregional therapies [10, 11, 13, 27-30]. Considering the achievement of early response and high response rate after combined TACE plus RT in the present study, a more potent locoregional therapy combined with RT should be preferentially considered in patients with liver-confined HCC accompanied by MVI.

Even though we observed high response rates after combined TACE plus RT in patients with HCC showing MVI, almost half of the patients did not respond to treatment. Moreover, the median PFS was 7.4 months in all patients, which indicates that patients with MVI experienced early tumor progression and still had very poor absolute survival rates. Subsequent systemic therapy (mostly sorafenib) was applied in $39.1 \%$ of patients who showed progressive disease after repeated TACE; however, the use of systemic therapy did not significantly affect patients' survival outcomes (Tables 3,4). In recent years, immune checkpoint inhibitors combined with molecular targeted agents have shown promising outcomes in treating advanced-stage HCCs [31]. RT showed both pro-immunogenic and immunosuppressive effects on immune 
response and induced a substantial increase in the expression of programmed cell death-1 and programmed cell death-ligand 1 in tumor cells [32]; further efforts such as RT combined with immune checkpoint inhibitors and novel systemic agents are required to improve the survival of patients with HCC having MVI. In addition, effective subsequent treatments after progression can also confer survival benefits, and are thus thought to be as important as first-line treatments for the prolongation of OS. Therefore, efforts should be made to develop more powerful treatments to overcome this unfavorable clinical situation.

The study has potential limitations. Due to the retrospective study design, the results may have been affected by selection bias or information bias. Even though we employed statistical methods including landmark and timedependent analyses to reduce the guarantee-time bias, there may be other biases that could not be controlled. In addition, we evaluated radiologic response as objectively as possible according to the mRECIST, but some subjective results might have been included because most patients had multiple HCCs and infiltrative tumor types at diagnosis. Tovoli et al. [33] showed that the decision on treatment response under sorafenib in HCC patients varied according to operators, especially in cases of nonspecially trained radiologists. In this study, to reduce the variability of response assessment, a well-trained radiologist and radiation oncologists independently reviewed the patient data, and a consensus decision was made in case of discrepancies. Finally, due to the retrospective design of the study, the follow-up imaging studies were not exactly performed at the fixed time points of 2 and 4 months, but rather within 2 weeks from the fixed time points. Despite the limitations stemming from the study design, the present study provides valuable information about the prognostic significance of radiologic responses by mRECIST in advanced-stage HCC showing MVI by including a large number of patients that were uniformly treated with TACE plus RT. In conclusion, we observed that the radiologic response rate assessed by mRECIST was a significant prognostic factor for OS in patients with advanced-stage HCC showing MVI after treatment with combined TACE plus RT.

\section{Statement of Ethics}

This study was approved by the Institutional Review Board of Asan Medical Center Seoul, Republic of Korea (IRB study No. 2017-0491), and the requirement for informed consent was waived due to the retrospective nature of the study.

\section{Conflict of Interest Statement}

The authors have no conflicts of interest to declare.

\section{Funding Sources}

This study was supported by a grant (\#2021IP0032-1) from the Asan Institute for Life Sciences of Asan Medical Center, Seoul, Republic of Korea.

\section{Author Contributions}

Jinhong Jung, Ji Hyeon Joo: conceptualization and design, funding acquisition, investigation, acquisition of data, formal analysis, methodology, statistical analysis, writing-original draft, and writing-review and editing. So Yeon Kim: investigation, formal analysis, methodology, and writing-review and editing. Jin Hyoung Kim: investigation, methodology, and writing-review and editing. Jonggi Choi: investigation, statistical analysis, methodology, and writingreview and editing. Danbi Lee: investigation, methodology, and writing-review and editing. Ju Hyun Shim: investigation, methodology, and writing-review and editing. Kang Mo Kim: investigation, methodology, and writing-review and editing. Young-Suk Lim: investigation, methodology, and writing-review and editing. $\mathrm{Han} \mathrm{Chu}$ Lee: investigation, methodology, and writing-review and editing. Jin-hong Park: investigation, methodology, and writing-review and editing. Sang Min Yoon: conceptualization and design, investigation, acquisition of data, formal analysis, methodology, supervision, writing-original draft, and writing-review and editing.

\section{Data Availability Statement}

The data that support the findings of this study are not publicly available due to the Personal Information Protection Act, but are available from the corresponding author upon reasonable request.

\section{References}

1 Bray F, Ferlay J, Soerjomataram I, Siegel RL, Torre LA, Jemal A. Global cancer statistics 2018: GLOBOCAN estimates of incidence and mortality worldwide for 36 cancers in 185 countries. CA Cancer J Clin. 2018 Nov;68(6):394-424.

2 Knox JJ, Cleary SP, Dawson LA. Localized and systemic approaches to treating hepatocellular carcinoma. J Clin Oncol. 2015 Jun 1; 33(16):1835-44.

3 European Association for the Study of the Liver. EASL clinical practice guidelines: management of hepatocellular carcinoma. J Hepatol. 2018 Jul;69(1):182-236.

4 Marrero JA, Kulik LM, Sirlin CB, Zhu AX, Finn RS, Abecassis MM, et al. Diagnosis, staging, and management of hepatocellular carcinoma: 2018 practice guidance by the American Association for the Study of Liver Diseases. Hepatology. 2018 Aug;68(2):723-50. 
5 Llovet JM, Ricci S, Mazzaferro V, Hilgard P, Gane E, Blanc JF, et al. Sorafenib in advanced hepatocellular carcinoma. N Engl J Med. 2008 Jul 24;359(4):378-90.

6 Cheng AL, Kang YK, Chen Z, Tsao CJ, Qin S, Kim JS, et al. Efficacy and safety of sorafenib in patients in the Asia-Pacific region with advanced hepatocellular carcinoma: a phase III randomised, double-blind, placebo-controlled trial. Lancet Oncol. 2009 Jan;10(1): 25-34.

7 Kudo M, Finn RS, Qin S, Han KH, Ikeda K, Piscaglia F, et al. Lenvatinib versus sorafenib in first-line treatment of patients with unresectable hepatocellular carcinoma: a randomised phase 3 non-inferiority trial. Lancet. 2018 Mar 24;391(10126):1163-73.

8 Bruix J, Cheng AL, Meinhardt G, Nakajima K, De Sanctis Y, Llovet J. Prognostic factors and predictors of sorafenib benefit in patients with hepatocellular carcinoma: analysis of two phase III studies. J Hepatol. 2017 Nov; 67(5):999-1008.

9 Finn RS, Qin S, Ikeda M, Galle PR, Ducreux M, Kim TY, et al. Atezolizumab plus bevacizumab in unresectable hepatocellular carcinoma. N Engl J Med. 2020 May 14;382(20): 1894-905.

10 Yoon SM, Lim YS, Won HJ, Kim JH, Kim $\mathrm{KM}$, Lee HC, et al. Radiotherapy plus transarterial chemoembolization for hepatocellular carcinoma invading the portal vein: longterm patient outcomes. Int $J$ Radiat Oncol Biol Phys. 2012 Apr 1;82(5):2004-11.

11 Yu JI, Park JW, Park HC, Yoon SM, Lim DH, Lee $\mathrm{JH}$, et al. Clinical impact of combined transarterial chemoembolization and radiotherapy for advanced hepatocellular carcinoma with portal vein tumor thrombosis: an external validation study. Radiother Oncol. 2016 Feb;118(2):408-15.

12 Korean Liver Cancer Association (KLCA); National Cancer Center (NCC), Korea. 2018 Korean Liver Cancer Association - National Cancer Center Korea practice guidelines for the management of hepatocellular carcinoma. Gut Liver. 2019 May 15;13(3):227-99.

13 Yoon SM, Ryoo BY, Lee SJ, Kim JH, Shin JH, An JH, et al. Efficacy and safety of transarterial chemoembolization plus external beam radiotherapy vs sorafenib in hepatocellular carcinoma with macroscopic vascular invasion: a randomized clinical trial. JAMA Oncol. 2018 May 1;4(5):661-9.

14 Kim YJ, Jung J, Joo JH, Kim SY, Kim JH, Lim YS, et al. Combined transarterial chemoem- bolization and radiotherapy as a first-line treatment for hepatocellular carcinoma with macroscopic vascular invasion: necessity to subclassify Barcelona Clinic Liver Cancer stage C. Radiother Oncol. 2019 Dec;141:95100.

15 Bruix J, Sherman M; American Association for the Study of Liver Diseases. Management of hepatocellular carcinoma: an update. Hepatology. 2011 Mar;53(3):1020-2.

16 Kim GA, Shim JH, Yoon SM, Jung J, Kim JH, Ryu MH, et al. Comparison of chemoembolization with and without radiation therapy and sorafenib for advanced hepatocellular carcinoma with portal vein tumor thrombosis: a propensity score analysis. J Vasc Interv Radiol. 2015 Mar;26(3):320-9.e6.

17 Chu HH, Kim JH, Shim JH, Yoon SM, Kim $\mathrm{PH}$, Alrashidi I. Chemoembolization plus radiotherapy versus chemoembolization plus sorafenib for the treatment of hepatocellular carcinoma invading the portal vein: a propensity score matching analysis. Cancers. 2020 Apr 29;12(5):1116.

18 Lencioni R, Llovet JM. Modified RECIST (mRECIST) assessment for hepatocellular carcinoma. Semin Liver Dis. 2010 Feb;30(1): 52-60.

19 Pan CC, Kavanagh BD, Dawson LA, Li XA, Das SK, Miften M, et al. Radiation-associated liver injury. Int J Radiat Oncol Biol Phys. 2010 Mar 1;76(3 Suppl):S94-100.

20 Giobbie-Hurder A, Gelber RD, Regan MM. Challenges of guarantee-time bias. J Clin Oncol. 2013 Aug 10;31(23):2963-9.

21 Prajapati HJ, Spivey JR, Hanish SI, El-Rayes BF, Kauh JS, Chen Z, et al. mRECIST and EASL responses at early time point by contrast-enhanced dynamic MRI predict survival in patients with unresectable hepatocellular carcinoma (HCC) treated by doxorubicin drug-eluting beads transarterial chemoembolization (DEB TACE). Ann Oncol. 2013 Apr; 24(4):965-73.

22 Gillmore R, Stuart S, Kirkwood A, Hameeduddin A, Woodward N, Burroughs AK, et al. EASL and mRECIST responses are independent prognostic factors for survival in hepatocellular cancer patients treated with transarterial embolization. J Hepatol. 2011 Dec;55(6):1309-16.

23 Vincenzi B, Di Maio M, Silletta M, D'Onofrio L, Spoto C, Piccirillo MC, et al. Prognostic relevance of objective response according to EASL criteria and mRECIST criteria in hepatocellular carcinoma patients treated with lo- co-regional therapies: a literature-based meta-analysis. PLoS One. 2015;10(7):e0133488.

24 Kudo M, Ueshima K, Chiba Y, Ogasawara S, Obi S, Izumi N, et al. Objective response by mRECIST is an independent prognostic factor for overall survival in hepatocellular carcinoma treated with Sorafenib in the SILIUS trial. Liver Cancer. 2019 Nov;8(6):505-19.

25 Meyer T, Palmer DH, Cheng AL, Hocke J, Loembé $A B$, Yen CJ. mRECIST to predict survival in advanced hepatocellular carcinoma: analysis of two randomised phase II trials comparing nintedanib vs sorafenib. Liver Int. 2017 Jul;37(7): 1047-55.

26 Lencioni R, Montal R, Torres F, Park JW, Decaens T, Raoul JL, et al. Objective response by mRECIST as a predictor and potential surrogate end-point of overall survival in advanced HCC. J Hepatol. 2017 Jun;66(6):1166-72.

27 Koo JE, Kim JH, Lim YS, Park SJ, Won HJ, Sung KB, et al. Combination of transarterial chemoembolization and three-dimensional conformal radiotherapy for hepatocellular carcinoma with inferior vena cava tumor thrombus. Int J Radiat Oncol Biol Phys. 2010 Sep 1;78(1):180-7.

28 Bang A, Dawson LA. Radiotherapy for HCC: ready for prime time? JHEP Rep. 2019 Aug; 1(2):131-7.

29 Zeng ZC, Fan J, Tang ZY, Zhou J, Qin LX, Wang JH, et al. A comparison of treatment combinations with and without radiotherapy for hepatocellular carcinoma with portal vein and/or inferior vena cava tumor thrombus. Int J Radiat Oncol Biol Phys. 2005 Feb 1;61(2): 432-43.

30 Kim DY, Park W, Lim DH, Lee JH, Yoo BC, Paik SW, et al. Three-dimensional conformal radiotherapy for portal vein thrombosis of hepatocellular carcinoma. Cancer. 2005 Jun 1; 103(11):2419-26.

31 Cheng AL, Hsu C, Chan SL, Choo SP, Kudo $\mathrm{M}$. Challenges of combination therapy with immune checkpoint inhibitors for hepatocellular carcinoma. J Hepatol. 2020 Feb;72(2): 307-19.

32 Choi C, Yoo GS, Cho WK, Park HC. Optimizing radiotherapy with immune checkpoint blockade in hepatocellular carcinoma. World J Gastroenterol. 2019 May 28;25(20):2416-29.

33 Tovoli F, Renzulli M, Negrini G, Brocchi S, Ferrarini A, Andreone A, et al. Inter-operator variability and source of errors in tumour response assessment for hepatocellular carcinoma treated with sorafenib. Eur Radiol. 2018 Sep;28(9):3611-20. 